\title{
DATUM TRANSFORMATION OF SPATIAL DATA AND APPLICATION IN CADASTRE
}

\author{
A. K1sa, B. Erkek, L. Ekin \\ General Directorate of Land Registry and Cadastre, Ankara, Turkey \\ tk36756@tkgm.gov.tr, berkek@tkgm.gov.tr, lutfiekin@gmail.com
}

\section{Commission IV, WG IV/1}

\author{
KEY WORDS: Datum, Datum Transformation, Cadastral Maps, Data Harmonization, Cadastre Web Services, Land Registry
}

\begin{abstract}
:
In Turkey, cadastral works have been started with local-based works in 1924 and speeded up after 1950's by using photogrammetry. Different measurement methods, coordinate systems and scales have been used in these works. As a result of primary cadastral activities two main products are generated; cadastral maps and title deeds. After this, cadastral data live on the maps, by cadastral activities carried out by cadastral offices and title deed data live on the registrations by land registration activities carried out by land registration offices. Up to 2005 different references systems such as local (graphic) and ED50 have been used for Cadastral maps production.
\end{abstract}

2000's Land Registry and Cadastre Information System (TAKBİS) Project has started as a pilot application by Land Registry and Cadastre (TKGM). After completion of pilot project spreading activities started in 2005 and still has been ongoing. On the other hand The government has taken the decision to finish primary cadastral activities within three years. The primary cadastral activities completed at the end of 2008. And also TKGM has completed metadata portal in 2008. At last, cadastral map updating (renovation) started in 2009 by using digital orthophoto with $30 \mathrm{~cm}$ GSD.

Today people have great expectations in accomplishing digital cadastral services, they need correct, reliable, easy and quick accessible land register and cadastral survey information. Even such request expressed in INPIRE directive by using ISO 191XX data standards. This means we have great hard work for spatial data conversion, datum and data transformation for map and cadastral data harmonization.

This paper presents results of investigation of used cadastral maps and used datums of the TKGM and possible transformation methods of datum and some recommendations for future applications.

\section{INTRODUCTION}

According to ISO TC 211 and OPEN GIS standards, "Coordinate Conversion" includes the mathematical relations of coordinates between same reference coordinate systems. "Coordinate Transformation", on the other hand, includes the relations of different reference coordinate systems. Coordinate transformation is named as "DATUM TRANSFORMATION" as well. In general, the primary concern of map users is the transformation between coordinates produced from the main reference coordinate system - ITRF "International Terrestrial Reference Frame" which was formed using space techniques and coordinates of existing networks.

According to ISO TC 211 and OPEN GIS standards, accuracy as data quality is defined as Network Accuracy. Network accuracy or absolute accuracy is the accuracy with regard to datum. This accuracy can be identified only by control surveying. In datum transformation, "Fitting Accuracy" has also to be calculated as an indication of integration between data and model. This value is the internal accuracy " $\mathrm{m} 0$ " and may not be concordant with network accuracy.

An accurate, reliable and persistent datum transformation depends on the parameters below:
1) The structure and accuracy of networks for both systems. Today, accurate, undistorted and homogenous position accuracies are obtained through GNSS technique. However, old networks produced by terrestrial techniques include many less accurate systematic effects. ITRF is managed as dynamic network, whereas old networks are managed in static structure.

2) The size of transformation area affects transformation accuracy and method. Therefore, obtaining different transformation accuracies using different methods for regional or local areas is in the possibility. In general, required accuracies can be obtained through evaluating very large and problematic areas in pieces. In the next step, persistence can be provided by combining these pieces.

3) The number of common points used in transformation and their distribution affects accuracy. For this reason, point density degree has to be as high as possible.

4) The mathematic and stochastic model used in datum transformation can be chosen with respect to the three factors above. The most important point to take into consideration while choosing the model is the possible systematic effects in network. These effects can be enumerated as crustal movements, edge measures in different scales etc. Some of the models in literature are enumerated below: 
Geometric Transformation Models (Helmert, Bursa-Wolf, Moledenski-Bedakes etc.), Two-parameter Polynomials, Finite Elements, Interpolation Methods (LSQ, Least Curvature, Triangulation etc.) (TKGM Turkish National Transformation Project Report 2008).

\section{GEODETIC INFRASTRUCTURE IN TURKEY}

After the adjustment of the National Fundamental Triangulation Network in 1954, the fact remains that some parts were revised, but there are errors in adjustment modeling related to the reduction of measurements; various plates move with different velocities because of tectonic properties in and around Turkey and these movements were not monitored systematically; because ED-50 coordinates were not renewed in accordance, points were displaced in different velocities and because the studies carried out before 1988 Regulation were in local coordinate systems, many engineering works including cadastre were done at local coordinate systems.

Since inner accuracy or local accuracy criteria were minded in densification works, information about network accuracies was not required. Therefore, detailed examination cannot be done. Moreover, the networks, formed according to 1988 Regulation, provide inner integrity. However, problems arise in larger scales. First and second order points were calculated with respect to adjustment, whereas lower degree points were calculated by inter/re-section methods.

Distortions resulting from crustal movements, landslides etc. in networks generated by conventional methods are an inevitable fact. Every single point group of these networks forms a separate datum. Up to a recent history in Turkey, ED-50 was used and all geodetic networks, maps and measures were generated at this datum. The National Geodetic Network studies in question were began in 1950-1954 by the General Command of Mapping (HGK) and 449,215 points were established with the subsequent densifications done by the cooperation of the General Directorate of Land Registry and Cadastre (TKGM) and HGK. These networks have an accuracy of 10-20 ppm. Third and fourth order 285,000 triangulation points were established, measured and calculated for the purpose of producing 1/5,000 scaled standard topographic maps. These points, whose 71,000 were produced by HGK and rest 215,000 by TKGM, are present within the archive of TKGM. The accuracy of the National Geodetic Network fell behind the accuracy that the modern technology has reached. In order to fulfil this need, the Turkish National Fundamental GPS Network (TUTGA), consisting of 594 points, was established between 1997-2001 by TKGM and HGK. The coordinates and velocities of TUTGA points were determined in ITRF. Relative accuracy of the network is $0.1-0.01 \mathrm{ppm}$, whereas point position accuracy is about $1-3 \mathrm{~cm}$ (TKGM Turkish National Transformation Project Report 2008).

\subsection{Some of the Results obtained between TUTGA and ED- 50}

a) \pm 3 -5 m transformation match was obtained for whole Turkey with 3D similarity transformation between TUTGA99A and ED-50 using 220 points.

b) $\pm 1.5-2 \mathrm{~m}$ transformation match was obtained for every single zone with separate similarity transformations. c) Similar results were obtained through modeling latitude and longitude differences.

d) For Izmir and Istanbul, transformation accuracies of $\pm 8 \mathrm{~cm}$ and $\pm 10 \mathrm{~cm}$ were obtained, respectively.

According to the $82^{\text {nd }}$ article of the Large Scale Map and Map Information Production Regulation, minimum transformation accuracy was taken as $\pm 9 \mathrm{~cm}$. According to the $100^{\text {th }}$ article, transformation enhancement and update tasks were given to TKGM, HGK and universities. However, increasingly growing municipality borders, Metropolitan municipalities and countrywide projects like TUSAGA-Aktif require horizontal and vertical parameters persistent for whole area with high network accuracy or transformation grid data. Using this data has to be juristically guaranteed. For example, technical and juridical guarantee has to be provided for transferring information produced at ED-50 or ITRF derived from legal cadastre information without accuracy loss. This requirement constitutes the primary concern of datum transformation.

All maps and coordinates produced countrywide until 2001 are on ED-50. There are around 283,000 map sheets on ED-50 within the body of TKGM. Transformation into ITRF datum of these map sheets is an inevitable fact. However, most of these map sheets are not digital and errors coming from production (erroneous measurement, drawing, edging etc.) exist. Bringing these maps into digital form with digitizing and absolute transformation will transfer the existing errors to digital map and result in legal consequences.

Transformation accuracy of ED-50 data and map sheet scale are desired to be concordant. Accuracy on 1/1,000 scaled map sheets is around $0.2-0.3 \mathrm{~m}$ and taking relative accuracy into consideration with respect to National Geodetic Networks, it is required to have common points at $10-30 \mathrm{~km}$ intervals whose coordinates are determined on ED-50 and ITRF. Thus, GNSS measurements will be required for a reasonable transformation at geodetic points that map sheets depend on and at produced details. This will be possible by loss in transformation accuracy (TKGM Turkish National Transformation Project Report 2008).

\section{COMMONLY USED DATUMS IN TURKEY}

\subsection{European Datum 1950 (ED-50)}

All maps and coordinates produced countrywide until 2001 are on ED-50. There are around 283,000 map sheets on ED-50 within the body of TKGM.

Up to a recent history in Turkey, ED-50 was used and all geodetic networks, maps and measures were generated at this datum. The National Geodetic Network studies in question were began in $1950-1954$ by HGK and 449,215 points were established with the subsequent densifications done by the cooperation of TKGM and HGK. These networks have an accuracy of 10-20 ppm. ED-50 represents International 1924 Ellipsoid which was defined locally for Europe (TKGM Turkish National Transformation Project Report 2008).

ED-50 takes International 1924 Ellipsoid $(a=6378388 \mathrm{~m}$; $\mathrm{b}=6356911.9461 ; \mathrm{f}=1 / 297 ; \quad \mathrm{e}=0.08199188998)$ as reference ellipsoid, and Greenwich Meridian as prime meridian. In this system, Helmertturm point $\left(\phi=52^{\circ} 22^{\prime} 51^{\prime \prime} .446 \mathrm{~N} ; \lambda=13^{\circ} 03^{\prime}\right.$ $58^{\prime \prime} .741 \mathrm{E}$; Geoid height $(\mathrm{N})=0 \mathrm{~m}$; north-south component of deflection of the vertical $(\zeta)=3 " .36$, east-west component of 
deflection of the vertical $\left.(\eta)=1^{\prime \prime} .78\right)$ in Potsdam/Germany is assumed to be the base point that the ellipsoid and geoid are coincident and the geodetic and astronomic coordinates are same. There is a shift up to a couple of hundred meters between the reference ellipsoid used in this system and the Earth's center of mass (Lenk and Firat, 2001).

\subsection{World Geodetic System 1984 (WGS-84)}

It is a terrestrial reference system used for GPS positioning by the US Department of Defense. Earth related satellite positions in GPS navigation message are announced in this system. WGS-84 is a conventional terrestrial reference system (NIMA, 1997).

WGS-84, at the same time, embodies physical properties of the earth gravity field.

It is a terrestrial system and its origin is the Earth's center of mass. $\mathrm{Z}$ axis is parallel to the mean Earth axis which was defined for 1984.0 epoch by the International Time Bureau $(\mathrm{BIH}) ; \mathrm{X}$ axis is the intersection of the Reference Longitude Plane defined by $\mathrm{BIH}$ and equatorial plane. $\mathrm{Y}$ axis is perpendicular to the $\mathrm{X}$ and $\mathrm{Z}$ axes at the Earth's center of mass in the manner of forming a right-hand system (Harita Forum, 2008).

\subsection{International Terrestrial Reference Frame (ITRF)}

In Turkey, ED-50 was used until 2001 and since then ITRF started to be used.

ITRF is a dynamic network which depends on a model assuming point coordinates, point movement velocities and all plates on Earth's crust move. It is a highly accurate reference network. ITRF is used to be able to eliminate deformations in national triangulation and leveling networks at application works, and benefit from GPS and new technologies at maximum level.

Since ITRF is updated at certain years, abbreviation is written as ITRFyy in the present day. "yy" represents last two digits of related year (P1rt1, 2005).

\section{STATUS OF THE GENERAL DIRECTORATE OF LAND REGISTRY AND CADASTRE}

Map sheets produced from the result of cadastral studies have the characteristics of infrastructure and preserve their state. Within the body of the General Directorate of Land Registry and Cadastre, map sheets using various methods (photogrammetric and terrestrial) have been produced from 1925 to the present day (TKGM Turkish National Transformation Project Report 2008).

\subsection{Photo Plans}

They are the $1 / 5,000$ scaled map sheets produced for the purpose of photogrammetric mapping by photogrammetric methods using air photos obtained at picture scale by removing the errors partially or putting to no direct evaluation. There are around 1800 map sheets of this type within the archive of TKGM. Parcels under a particular size limit are measured and then transferred to these maps with the values. Maps are used as base plate for both restriction and detection studies. Digitization of these maps has been done by directorates for 5-6 years and values obtained from digitization results are used only for area calculation. Points obtained by the result from digitization of these maps alter with respect to scanning and application errors and this error rate is not less than 5-6 $\mathrm{m}$ in every point. Besides, the scale difference between the middle and corner of the image, rectification errors and interpretation errors are added on as well. Even it is claimed that scanning old images by a high resolution scanner and evaluating these scanned images by digital photogrammetric methods result in obtaining good values, there is a fact that error of these values is $1,5-2 \mathrm{~m}$, and interpretation and digitization errors are added on as well.

\subsection{Map Sheets Produced by Photogrammetric Method}

They are the map sheets which are obtained by the help of transformation of the reasonably distributed ground control points on flight area, after the evaluation of approximately $1 / 16,000$ scaled air photos obtained at the image scale in the purpose of photogrammetric map production by either analogue or digital methods. The most accurate result could be $\pm 2 \mathrm{~m}$ from the numeric values obtained by reading coordinates from line maps.

\subsection{Map Sheets Produced in Local Coordinate System}

They are the map sheets which are produced with respect to $2 \mathrm{D}$ plane coordinate system by accepting any starting point as origin. Best values produced by this method contain detail measurements done at ground control points generated using zoning coordinates. Line maps produced by this method are with local quality and the accuracy of produced coordinate values alters with respect to benchmark accuracy and talent and devotion of surveyor. As map sheets produced by this method are with local quality, they cannot be combined with other local network. In case they are combined, serious technical errors and non-recoverable legal consequences appear. In these maps, lack of structural measurement, uncontrolled measurement, edging and misrepresentation of ground exist apart from many error sources.

\subsection{Map Sheets Produced by Graphic Method}

They are the cadastral line map sheets which do not have coordinate information and grid network on it and produced by terrestrial surveying method. Most of map sheets were produced by this method until 1970. Map sheets produced by this method contain the most valuable places of residential areas. The biggest disadvantages of these map sheets are absence of ground control points on ground at application, hugeness of translation and rotation in $\mathrm{x}$ and $\mathrm{y}$ directions, irregularity of translation and rotation at these points, lack of translation and rotation quantity knowledge, edging with neighbor map sheets problem and having minimum point position accuracy of 2-3 m. As indicated above, since these map sheets are applied at large cities' centers and despite renewing most of these places through town planning and because of arising a huge increase in parcel value at remaining areas, these parcels cause serious troubles to implementers. In addition to changing error source with respect to each surveyor, errors such as lack of structural measurement, uncontrolled measurement, drawing and area calculation errors, edging and misrepresentation of ground exist.

4.5 Map Sheets Produced based on National Coordinate System (ED-50) 
They are the map sheets which are produced with numeric or line measurement values with the scale of $1 / 1,000$ at residential areas, or the scale of $1 / 2,000$ at non-residential areas if there is no photogrammetric map during facility cadastre. In general, densification is done with intersection points and string triangulation starting from first, second and third order triangulation points during the production of these map sheets. The accuracy of ground control point at map sheets produced by this method affects whole detail points. Since produced map sheets are opened in UTM projection system with respect to full map sheet system, in case there is a possible error, edging with borders produced at neighbor unit is never obtained. In this case, empty or overlapping areas are seen. With this method, prismatic survey was done at residential areas whereas classic tacheometric survey was done at non-residential areas, until the use of electronic tacheometers. Once again, as operator talent and measurement accuracy are important at measurements done in these areas, numeric values may correspond to erroneous spots in some regions.

\subsection{Numeric Method based on ITRF}

They are the studies which are done via tendering procedure within the context of law numbered 3402-5304. As GPS is used actively in this process, only random errors can be in question. This type of errors can be overcome easily within the system (TKGM Turkish National Transformation Project Report 2008).

\section{CURRENT STATUS AND PROBLEMS ENCOUNTERED}

In Turkey, there are map sheets on various datums produced by either TKGM or another institutions and organizations. Problems encountered on these map sheets can be grouped under two categories.

\subsection{Integration Problems on Map Sheets Produced at Same Datum}

Despite the fact that map sheets produced at same datum generally have inner consistency, they have a frequently encountered issue which is being full of random and systematic errors. It is very hard to detect such errors. For example, dependency of control points established in a region to route, inner consistency of detail points measured from that route; however, drift in the level of meters when generated from other routes. Besides, skipping necessary reductions during the production of control points can make the network consistent in itself, but will make inconsistent in global sense. For example, Araklı and Sürmene are two neighbor districts of Trabzon City. For these cities, there are map sheets produced at both ED-50 and the local datum in the past years. Under normal conditions, border map sheets of these two cities have to have edging property. However, edging does not occur in real or an empty buffer zone is seen between the two districts or overlapped parcels are seen.

\subsection{Integration Problems on Map Sheets Produced at Different Datums}

Map sheets produced at different datums are the biggest problem. In this type of map sheets, inner random/systematic errors preclude association with a map sheet produced at same system as well as integration with a different datum not surprisingly. The problem would be bigger if the system was produced with one of the methods from local, national or graphic. For instance, there are three working areas which are produced with local, national or graphic methods one by one. Under normal conditions, edging will never be obtained at these three map sheets and different transformation parameters will be applied (TKGM Turkish National Transformation Project Report 2008).

\section{SUGGESTIONS AND RESULTS}

Problems given in general under the two subtitles above can be solved by the suggestions and methods indicated below (TKGM Turkish National Transformation Project Report 2008).

1) Point sets assumed to be on ED-50, having inner consistency but containing gross errors when considered in network structure and distortions arisen from partly erroneous projection reductions should be determined using a proper test technique and if they have a systematic effect, they should be embodied in a new point set through re-modeling, which has an appropriate distribution character. Proper point sets should be determined by both additional and available measurements to be able to make a new modeling.

2) Measurements on various reference epochs (1998.00, 2000.45, 2005.00 and new epochs oncoming) of ITRF datum should be transferred to a common epoch. All the necessary data is present within the archive of TKGM.

3) Making legal arrangement in the article of Legal Law, which is the biggest obstacle, with regard to a report obtained after doing an adequate technical research on a suitable background will be more effective. In order to prevent misapplications here such as loss of a right, second cadastre formulae whose flexibility are not opened to interpretation should definitely be searched and remedies for this consideration should be sought with respect to legal background, technical aspect and resource procurement of this work. Moreover, this remedy should be applied in multidirectional cadastre as indicated in 2014 cadastre expansion.

4) Reliability of points used in ED-50 - ITRF transformations done by TKGM up to now should be re-examined and inner confidence standards should be well determined. Besides, it is obvious that stable establishments will highly enlighten the transformation. Studies done by various methods should primarily be examined and concordance to each other should be researched. Moreover, it should be found out that how much part of the base plates can be saved. While carrying out this work, field test measurements should certainly be done and results should be acquired in a way to generate accurate data. This accurate statistical data should be quickly finalized and relevant decisions should be taken and performed.

5) Cadastral base plates produced by cadastre organization should absolutely be passed through control mechanism. With this study, it is realized that transformation of graphic cadastral base plates to digital form is quite hard and impossible to perform for all areas. Particularly, it is observed that thinking digitization process in cadastral block scale instead of map sheet scale is more convenient, because different cadastral blocks in same map sheets can include both consistent and inconsistent cadastral blocks. In practical terms, consistent cadastral blocks can be converted to digital form, yet re-cadastre should definitely be done for the rest. 
6) To be able to set up a Spatial Information System (Field, City, and Geographic Information Systems etc.), map and cadastre information produced from national coordinate system and valid in legal should be used in the same way, the ones that are able to be converted from existing data to national coordinate system with the necessary accuracy that system requires should be used after transformation and if the ones that do not carry these properties require enhancement, then they should be added to system after their regeneration (TUJK, 2008).

\section{REFERENCES}

Harita $\quad$ Forum, 2008 www.haritaforum.com/index.php/topic,114.msg231.htm\#msg23 1 (25 Dec. 2008).

Lenk, O., and Firat, O., 2001. Turkish National Fundamental GPS Network 1999 (TUTGA99), Technical Report, General Command of Mapping, Ankara.

NIMA (National Imagery and Mapping Agency), 1997. World Geodetic System 1984: Its Definition and Relationships with Local Geodetic Systems, NIMA TR8350.2.1997.

Purt1, A., 2005. An Application for Comparison between Control Points (Benchmarks) Generated with Conventional Methods and GPS Coordinates, Chamber of Surveying and Cadastre Engineers, 2005/2-93.

TUJK (Turkish National Geodesy Commission), 2009. Coordinate Systems, Geoinformation and Land Management Bulletin, 2009/1-100.

Turkish National Transformation Project Report, 2008. Ministry of Public Works and Settlement, General Directorate of Land Registry and Cadastre, Department of Photogrammetry and Geodesy, Ankara. 